\section{PSYCAUSE}

Revue scientifique étudiante de

l'École de psychologie de l'Université Laval
UNIVERSITÉ LAVAL

Faculté des sciences sociales École de psychologie

SEPTEMBRE 2018 - VOL. $8 \mathrm{~N}^{\circ} 2$

\title{
LES CONSÉQUENCES D'UTILISER DIFFÉRENTES APPROCHES DE MESURE DE L'IMPLICATION PATERNELLE
}

Nicolas R.-TURGEON ${ }^{1}$ *, Roxanne PARIS-BLAIS ${ }^{1}$, Cynthia MARTINEAU-HOUDE¹,

Catherine LEVESQUE ${ }^{1} \&$ Célia MATTE-GAGNÉ ${ }^{1}$

1 École de psychologie, Université Laval, Québec, Canada

*nicolas.r-turgeon.1@ulaval.ca

\section{Pour citer l'article}

R.-Turgeon, N., Paris-Blais, R., Martineau-Houde, C., Levesque, C., \& Matte-Gagné, C. (2018). Les conséquences d'utiliser différentes approches de mesure de l'implication paternelle. Psycause : Revue scientifique étudiante de l'École de psychologie de l'Université Laval, 8(2), 21-23. 


\title{
LES CONSÉQUENCES D'UTILISER DIFFÉRENTES APPROCHES DE MESURE DE L'IMPLICATION PATERNELLE
}

\author{
Nicolas R.-TURGEON, Roxanne PARIS-BLAIS, Cynthia MARTINEAU-HOUDE, Catherine \\ LEVESQUE \& Célia MATTE-GAGNÉ \\ École de psychologie, Université Laval, Québec, Canada
}

\section{Introduction}

De plus en plus d'études suggèrent que l'implication paternelle est bénéfique pour le développement de l'enfant (Lamb, 2010; Lamb \& Lewis, 2013; Larzelere, Morris \& Harrist, 2013; Sarkadi, Kristiansson, Oberklaid \& Bremberg, 2008). Ainsi, l'étude des facteurs qui permettent de promouvoir l'implication paternelle revêt une importance particulière (Pleck, 2012). La conceptualisation de l'implication paternelle et les outils utilisés pour mesurer ce construit varient toutefois d'une étude à l'autre ce qui rend difficile une synthèse de la littérature dans le domaine (Turcotte, Dubeau, Bolté \& Paquette, 2001).

Selon Lamb, Pleck, Charnov et Levine (1987), l'implication paternelle se définit comme le degré d'engagement, d'accessibilité et de responsabilité du père à l'égard de son rôle parental. Ce degré peut être mesuré à l'aide de différents outils. Ceux-ci se regroupent essentiellement en deux catégories: (a) les questionnaires de fréquences (QF); et (b) les journaux de bord temporel (JBT). Les QF mesurent la fréquence $d^{\prime}$ implication du père dans les activités parentales quotidiennes, alors que les JBT mesurent le temps que le père passe à faire des activités avec ou pour son enfant. Dans la littérature, les chercheurs utilisent de façon interchangeable ces deux outils pour mesurer l'implication paternelle, et ce, malgré le fait qu'ils possèdent des caractéristiques distinctes susceptibles d'entraîner des variations dans la relation entre l'implication paternelle et les construits examinés. Comme ces outils n'ont jamais été mis en relation, il est difficile de savoir s'ils mesurent exactement le même construit. Des divergences sont d'ailleurs observées dans la relation entre l'implication paternelle et l'alliance parentale, en fonction de l'approche de mesure utilisée (JBT ou QF). Pour mieux comprendre les conséquences d'utiliser différentes approches de mesure de l'implication paternelle, cette étude examine pour la première fois les relations entre les estimés d'implication paternelle obtenus à l'aide d'un QF et d'un JBT et les relations entre ces estimés et l'alliance parentale, un prédicteur reconnu de l'implication paternelle.

\section{Méthode}

Quatre-vingt-six pères ayant un enfant âgé de 6 mois et demeurant dans la ville de Québec avec la mère de leur enfant (familles biparentales intactes) ont été recrutés pour participer à cette étude. L'implication des pères a été mesurée à l'aide d'un JBT et d'un QF. Le JBT a été administré sous la forme d'une entrevue semistructurée réalisée au domicile des participants. Dans le cadre de cette entrevue, les pères étaient invités à décrire leur routine quotidienne, par tranche de 30 minutes, du lundi au dimanche. Pour chaque tranche de 30 minutes, l'expérimentateur devait indiquer sur une grille horaire, si le père effectuait une activité avec ou pour l'enfant (p. ex., changer la couche ou préparer les repas). Le nombre de 30 minutes $d^{\prime}$ implication par semaine était ensuite calculé afin de former un score global d'implication paternelle. À la fin de la rencontre, les pères recevaient un lien électronique pour compléter le QF et I'Inventaire d'Alliance Parentale (IAP). Dans le $Q F$, le père devait indiquer sur une échelle allant de (1) = jamais à (6) = chaque jour, à quelle fréquence il participe généralement 
aux 19 activités parentales décrites (p. ex., changer la couche). Dans I'IAP, composé de 20 items, le père devait indiquer sur une échelle de type Likert allant de (1) = fortement en désaccord à (5) = fortement en accord son degré de coopération et d'engagement avec sa conjointe dans l'éducation de l'enfant.

\section{Résultats et discussion}

Les résultats des analyses corrélationnelles effectuées montrent que la fréquence et la durée $d$ 'implication du père sont significativement et modérément associées $(r=0,40 ; p<0,001)$. Les résultats des analyses de régression démontrent quant à euxqu'en contrôlant pourle sexe de l'enfant, le nombre d'années de scolarité du père, son nombre d'heures de travail et son revenu annuel, l'alliance parentale prédit significativement la fréquence $d$ 'implication paternelle $(\beta=0,25 ; p=0,024)$, mais pas la durée d'implication.

Les résultats suggèrent que les $Q F$ et les JBT ne mesurent pas exactement les mêmes dimensions de l'implication paternelle et que ces dimensions ne sont pas prédites par les mêmes facteurs. Les pères qui s'impliquent plus fréquemment dans les activités parentales quotidiennes passent généralement plus de temps avec leur enfant, toutefois la relation modérée entre la fréquence et la durée d'implication suggère que les pères qui s'impliquent le plus en termes de fréquence ne sont pas toujours ceux qui s'impliquent le plus en termes de temps. En ce qui concerne le deuxième objectif de l'étude, les résultats indiquent que les pères qui rapportent former une bonne alliance parentale avec leur conjointe s'impliquent plus fréquemment dans les activités parentales quotidiennes, mais ne passent pas plus de temps avec leur enfant. Ces résultats sont cohérents avec ceux des études antérieures qui ont utilisé une seule approche de mesure. II se pourrait que pour maintenir une bonne alliance avec la mère, le père s'implique quotidiennement dans les activités parentales, sans toutefois passer beaucoup de temps avec son enfant. La durée de l'implication paternelle pourrait s'expliquer davantage par des facteurs propres à l'enfant, tels que son tempérament ou sa motivation à participer aux activités proposées par le père, mais cela demeure à investiguer. Cette étude met en lumière les divergences que l'utilisation de différents outils de mesure de l'implication paternelle peut entraîner dans les résultats des études réalisées dans le domaine. Ces divergences soulignent l'importance d'interpréter et de nuancer les résultats des études portant sur l'implication paternelle à la lumière des choix méthodologiques effectués.

\section{Références}

Lamb, M. E. (2010). How do fathers influence children's development? Let me count the ways. Dans M. E. Lamb (Ed.), The role of the father in child development ( $5^{\mathrm{e}}$ éd., pp. 1-26). Hoboken, NJ: John Wiley \& Sons Inc.

Lamb, M. E., \& Lewis, C. (2013). Father-child relationships. Dans N. J. Cabrera, \& C.S.Tamis-LeMonda(Eds.), Handbookoffather involvement: Multidisciplinary perspectives (2e éd., pp. 119-135). New York, NY: Taylor \& Francis.

Lamb, M. E., Pleck, J. H., Charnov, E. L., \& Levine, J.A. (1987). A biosocial perspective on paternal behavior and involvement. Dans J. B. Lancaster, J. Altmann, A. S. Rossi, \& L. R. Sherrod (Eds.), Parenting across the life span: Biosocial dimensions (pp. 111-142). Hawthorne, NY: Aldine.

Larzelere, R. E., Morris, A. S., \& Harrist, A. W. (2013). Authoritative parenting: Synthesizing nurturance and discipline for optimal child development. Washington, DC: American Psychological Association. Pleck, J. H. (2012) Integrating father involvement in parenting research. Parenting, 12, 243-253. doi:10.10 80/15295192.2012.683365 
Sarkadi, A., Kristiansson, R., Oberklaid, F., \& Bremberg, S. (2008). Fathers' involvement and children's developmental outcomes: A systematic review of longitudinal studies. Acta paediatrica, 97, 153-158. doi:10.1111/ j.1651-2227.2007.00572.x

Pleck, J. H. (2012) Integrating father involvement in parenting research. Parenting, 12, 243253. doi:10.1080/15295192.2012.683365
Turcotte, G., Dubeau, D., Bolté, C., \& Paquette, D. (2001). Pourquoi certains pères sont-ils plus engagés que d'autres auprès de leurs enfants? Une revue des déterminants de l'engagement paternel. Revue canadienne de psychoéducation, 30, 65-93. Récupéré sur https://unites.uqam.ca/grave/prospere/ pages/pdf/Turcotte_Dubeau.pdf

\section{LIENS ENTRE LA SOLITUDE ET LES TYPES D'UTILISATION DE FACEBOOK}

Mélissa BAILLARGEON, Anne-Hélène HARRISSON, Joanie MERCIER \& Marie-Claude RICHARD École de psychologie, Université Laval, Québec, Canada

\section{Introduction}

Plusieurs travaux ont tenté de clarifier les liens entre la solitude et l'utilisation des réseaux sociaux en ligne sans parvenir à un consensus. Par exemple, les études de la méta-analyse de Song et coll. (2014)—qui s'intéressent à la quantité de temps passé sur Facebook, à l'usage excessif ou compulsif de Facebook, et celles comparant les utilisateurs aux nonutilisateur de Facebook-tendent à montrer un lien avec une augmentation de la solitude. Par contre, d'autres études de cette même méta-analyse tendent à montrer un lien entre I'intensité de l'utilisation de Facebook (c.-à-d. le nombre d'amis, le temps quotidien passé sur Facebook, la quantité de contenu créé, le nombre de communications directes entretenues sur Facebook et leur attitude par rapport à Facebook) et une diminution de la solitude. Une explication de ces résultats divergents est que les études ne prennent pas en compte les types d'utilisation de Facebook (c.-à-d. actif social, actif non social et passif; voir le Tableau 1 tiré de Gerson, Plagnol \& Corr, 2017). De plus, certains auteurs ont exploré la relation entre les types d'utilisation de Facebook et la perception de soutien social (Seo, Kim \& Yang, 2016). II ressort donc de ce qui précède que les personnes utilisant davantage Facebook de façon active ont une plus grande perception de soutien social que ceux utilisant les autres types d'utilisation. Les objectifs de cette étude consistent à déterminer si : (a) les types d'utilisation de Facebook sont associés au sentiment de solitude; et si (b) la perception de soutien social contribue à expliquer cette relation.

\section{Méthode}

Trois cent onze adultes québécois utilisateurs de Facebook âgés de 18 à 29 ans $(M=22,59 ; \dot{E}-T=$ 2,68 ) ont rempli le questionnaire de 41 questions à partir de l'application web LimeSurvey.

L'Échelle de solitude de l'Université Laval (ÉSUL; de Grâce, Joshi \& Pelletier, 1993), la version francophone du UCLA Loneliness Scale, a été utilisée pour évaluer la solitude. Cette échelle comprend 20 énoncés autodescriptifs pour lesquels le participant indique la fréquence d'apparition d'un sentiment spécifique de solitude dans sa vie (Russell, 1996). La souséchelle Soutien familial du Multidimensional Scale of Perceived Social Support (MSPSS), adaptée par Frison et Eggermont (2016), a été utilisée pour évaluer la perception de soutien social sur Facebook (Zimet, Powell, Farley, Werkman \& Berkoff, 1990). Le Passive and Active Use Measure (PAUM; Gerson et coll., 2017) a été utilisé pour évaluer les types 